\title{
Automatic Track Inspection in Railway Network
}

\author{
S Ramesh ${ }^{1}$, S Arvind ${ }^{2}$, Rituraj $^{3}$, Mukesh Kumar Roy $^{4}$ \\ ${ }^{I}$ (Asst. Prof., Dept. of EEE, SRM University, India) \\ $2,3,{ }^{4}$ (B.Tech, Dept. of EEE, SRM University, India
}

\begin{abstract}
In India rail transport occupies a prominent position in providing the necessary transport infrastructure to sustain needs of a rapidly growing economy. Today, India possesses the fourth largest railway network in the world. However, in terms of the reliability and safety parameters, we have not yet reached truly global standards. The main problem about a railway analysis is detection of cracks in the structure. If these deficiencies are not controlled at early stages they might lead to a number of derailments resulting in a heavy loss of life and property. This project proposes a cost effective solution to the problem of railway track crack detection utilizing RF control assembly which tracks the exact location of faulty track which then mended immediately so that many lives will be saved.
\end{abstract}

Keywords: Automation, CAN Controller, Cracks, Detection, GSM, GPS

\section{Introduction}

Indian Railways is an Indian state-owned enterprise, owned and operated by the Government of India through the Ministry of Railways. India possesses fourth largest network in the world exceeded only by those of the United States, Russia and China. The Indian railway network stretches across the globe with a length of 115,000 kilometers $(71,000 \mathrm{mi})$ over a route of 65,000 kilometers $(40,000 \mathrm{mi})$ covering 7,500 stations. The network traverses every nook and cranny of the nation carries over 25 million passengers and 2.8 million tons of freight daily. Railways were first introduced to India in 1853 from Bombay to Thane. In 1951, the systems were nationalized as one unit, the Indian Railways, becoming one of the largest networks in the world. There are various types of trains in India like Rajdhani, Shatabdi, Duronto, Garib Rath, Janshatabdi, Express trains, Goods trains and Sub-Urban trains. As per the electric traction is concerned, a total of 23,541 km was electrified by $31^{\text {st }}$ March 2013, mostly on heavy density routes and in next few years the whole Indian railway network will be electrified. There are two causes of cracks in rail tracks i.e. Natural and Artificial. Natural causes are like weather, floods, cyclone, landslides etc. Artificial causes are like terrorist attacks etc. Though the rail transport in India is growing at a rapid pace, the associated safety infrastructure are not up to international standards. Our facilities are inadequate compared to the international standards and as a result, there have been frequent derailments that have resulted in severe loss of valuable human lives and property as well. On further analysis of the factors that cause these rail accidents, recent statistics reveal that, between year 2000 and 2013, more than 30 derailments took place of which approximately $90 \%$ are due to cracks on the rails. These accidents results in thousands of death and leaving lakhs of people injured. The principal problem has been the lack of cheap and efficient technology to detect problems in the rail tracks and of course, the lack of proper maintenance of rails which have resulted in the formation of cracks in the rails and other such problems caused by anti-social elements which jeopardize the security of operation of rail transport. So in this proposed model we are giving the solution for the detection of cracks.

Other techniques based on ultrasonic, electromagnetic waves and LED-LDR also contributed to the detection scheme but they can investigate only the core of the track rather than checking for surface cracks and the surfaces where maximum faults are located. Our design is based on automatic crack detecting engines using RF module with track side node.

\subsection{Microcontroller}

\section{Components Of The Surveying System}

The AT89S52 is a low-power, high performance CMOS 8-bit microcontroller with 8K bytes of insystem programmable Flash memory. The device is manufactured using Atmel's high-density nonvolatile memory technology and is compatible with the industry-standard 80C51 instruction set and pin out.

The AT89S52 provides the following standard features: 8K bytes of Flash, 256 bytes of RAM, 32 I/O lines, Watchdog timer, two data pointers, three 16-bit timer/counters, a six-vector two-level interrupt architecture, a full duplex serial port, on-chip oscillator and clock circuitry. The AT89S52 provides a highlyflexible and cost-effective solution to many embedded control applications. 


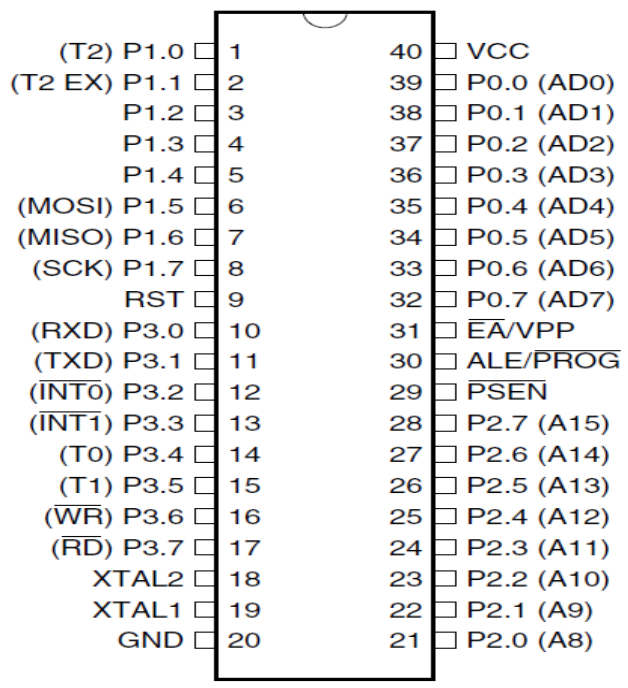

\subsection{Global Positioning System (GPS)}

Fig. 1. ATMEL Processor

The Global Positioning System (GPS) is a space-based satellite navigation system that provides location and time information in all weather conditions, anywhere on or near the Earth where there is an unobstructed line of sight to four or more GPS satellites. The GPS project was developed in 1973 by the U.S. Department of Defense (DOD) and was originally run with 24 satellites. It became fully operational in 1995. Each satellite continually transmits messages that include-

- The time the message was transmitted.

- Satellite position at time of message transmission.

The receiver uses the messages it receives to determine the transit time of each message and computes the distance to each satellite using the speed of light. In our design, the purpose of GPS is to find the exact location of the fault.

\subsection{Global System for Mobile communication (GSM)}

Global System for Mobile Communications is a standard developed by the European Telecommunications Standards Institute (ETSI) to describe protocols for second generation (2G) digital cellular networks used by mobile phones. GPS is an open, digital cellular technology used for transmitting mobile voice and data services.

GSM networks operate in a number of different carrier frequency ranges, with most 2G GSM networks operating in the $900 \mathrm{MHz}$ or $1800 \mathrm{MHz}$ bands. The longest distance the GSM specification supports in practical use is 35 kilometers (22 mi). GSM- R, Global System for Mobile Communications-Railway or GSM-Railway is an international wireless communications standard for railway communication and applications. A sub-system of European Rail Traffic Management System (ERTMS), it is used for communication between train and railway regulation control centers. In our design, the purpose of the GSM is to send alert signal to the control room.

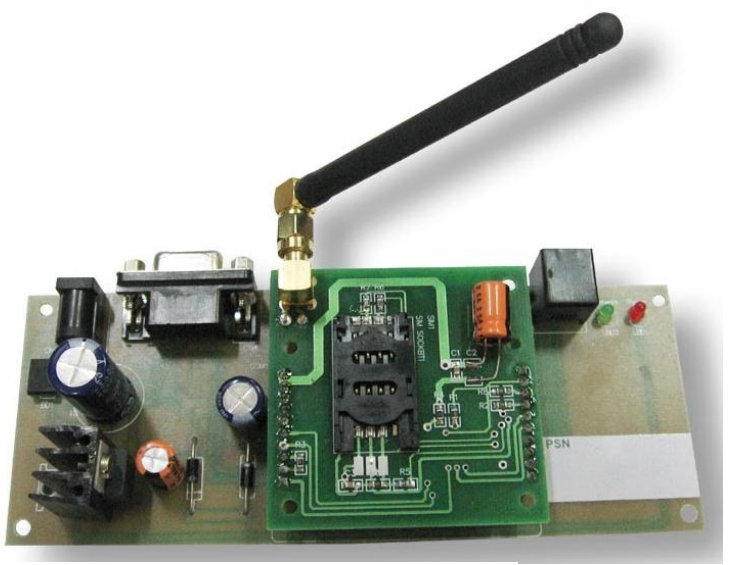

Fig. 2. GSM Module 


\subsection{CAN Controller}

Controller area network is a vehicle bus standard designed to allow microcontrollers and devices to communicate with each other within a vehicle without a host computer. CAN bus is a message-based protocol, designed specifically for automotive applications but now also used in other areas such as aerospace, maritime, industrial automation and medical equipment. Development of the CAN bus started originally in 1983 at Robert Bosch GmbH. The protocol was officially released in 1986 at the Society of Automotive Engineers (SAE) congress in Detroit, Michigan. The first CAN controller chips, produced by Intel and Philips, came on the market in 1987. Bosch published the CAN 2.0 specification in 1991.

\subsection{RF Transmitter with Encoder}

The RF module, as the name suggests, operates at Radio Frequency. The corresponding frequency range varies between $30 \mathrm{kHz} \& 300 \mathrm{GHz}$.

The Features of the HT-12E Encoder are Operating voltage: $2.4 \mathrm{~V} \sim 12 \mathrm{~V}$ for the HT12E and the Low power and high noise immunity. It has positive polarity and Minimal external components and 18-pin DIP/20pin SOP package.

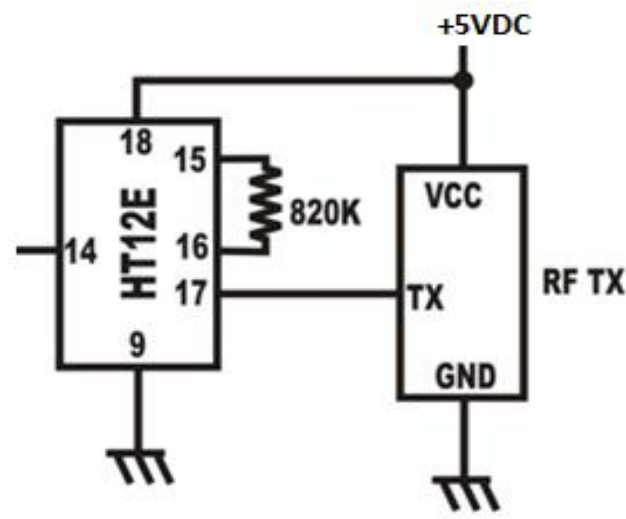

Fig. 3. RF Transmitter with Encoder

Transmission through RF is better than IR because of many reasons. Firstly, signals through RF can travel through larger distances making it suitable for long range applications. Also, while IR mostly operates in line-of-sight mode, RF signals can travel even when there is an obstruction between transmitter \& receiver. Next, RF transmission is more strong and reliable than IR transmission. RF communication uses a specific frequency unlike IR signals which are affected by other IR emitting sources. In our design, the purpose of RF transmitter is to transmit signal from the node to the receiver section installed in the incoming train.

\subsection{RF Receiver with Decoder}

The RF transmitter/receiver operates at $433.92 \mathrm{MHz}$, and has a sensitivity of $3 \mathrm{uV}$. The receiver operates from 4.5 to 5.5 volts-DC, it has both linear and digital outputs.

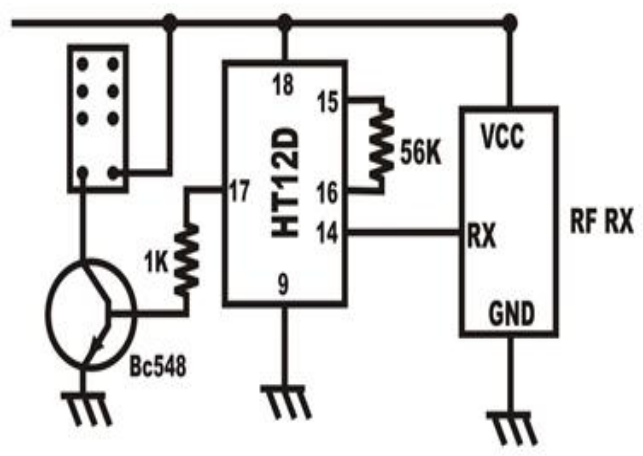

Fig. 4. RF Receiver with Decoder

The features of the HT-12D Decoder Operating voltage: $2.4 \mathrm{~V} \sim 12 \mathrm{~V}$ and it has Low power and high noise immunity. It has made in CMOS technology with Low standby current and also Capable of decoding 12 bits of information. Built-in oscillator needs only 5\% resistor with valid transmission indicator. Easy interface with an RF transmission medium and Minimal external components and 18-pin DIP/20-pin SOP package. In our design, the purpose of RF receiver is to receive the signal transmitted by the RF transmitter installed in the node side. 


\subsection{LCD Display}

A liquid-crystal display (LCD) is a flat panel display, electronic visual display, or video display that uses the light modulating properties of liquid crystals. This LCD display will be installed in the train to alert the driver when the crack is detected.

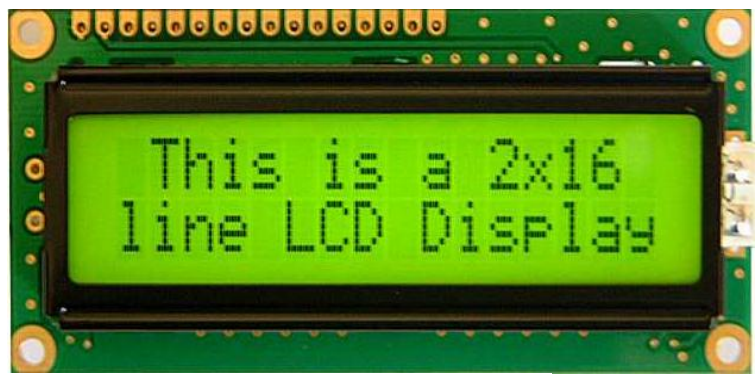

Fig. 5. LCD Display

\section{Nature Of Work}

The Indian railway network is the biggest rail network in south Asia and perhaps the most complicated in all over the world. There are so many different types of train's such as local, fast, passenger, goods etc. and their so many multiple routes. Our design is based on automation engines with track side node. The track side node is mounted to the post which is located every $300 \mathrm{~m}$. Here we can use the electrified lamp as post, because nowadays all routes are mostly electrified. According to our analysis, for every $300 \mathrm{~m}$ there is a post called electrified lamp. Devices like GPS, GSM and RF transmitter will be installed in these nodes. In trains, we will fix a device in front of the engines. This device will generate RF rays or RF current. So engines should pass the rays or current in the tracks. RF transmitter will also be installed in the train engine to receive signal. The post is connected to the tracks also. The automated output is connected to the RF rays.

When the train gets started, the rays also starts and it will be splashing in the tracks above 1 kilometer. The reason we are using rays that splashes above 1 kilometer is because there are different types of trains available like Rajdhani, Duronto, Janshatabadi, Super Fast, express trains, goods train and all are having different speed conditions. In the track side node, it is based on the electrified lamp in which we fix GSM, GPS and RF transmitter on that node. Node is nothing but every 4 post interconnected to make the node. So when the train get starts, the rays will also starts. For example, let us consider a route of length 3 kilometers. So when the train starts, the rays will splash above 1 kilometer, and there are electrical post installed at every $300 \mathrm{~m}$ distance. Incase if there is no cracks in between the first two post, the rays will pass through the next post without any disturbances. If there is a crack between 2nd and 3rd post, the rays will not pass through the next post within the particular duration, then the GSM will become active and it will send the message to the nearest control room along with the longitudes and latitudes of the fault location calculated by the GPS installed. Simultaneously, the $\mathrm{RF}$ transmitter connected in the node will also send alert signal to the RF receiver installed in the incoming train and the output will be displayed in the LCD display.

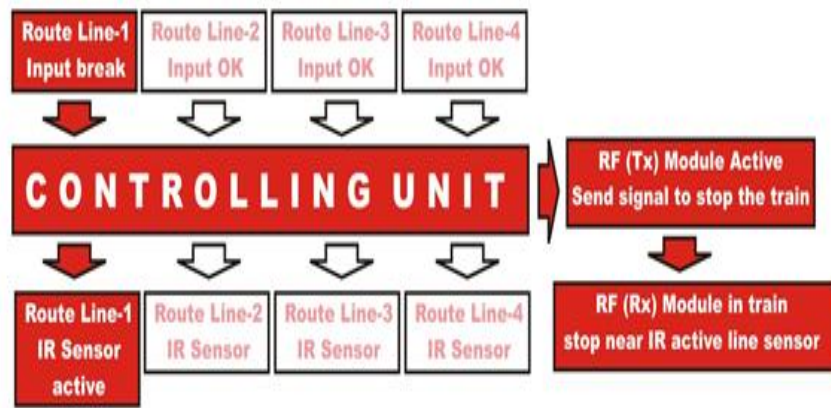

Fig. 6. Block Diagram 


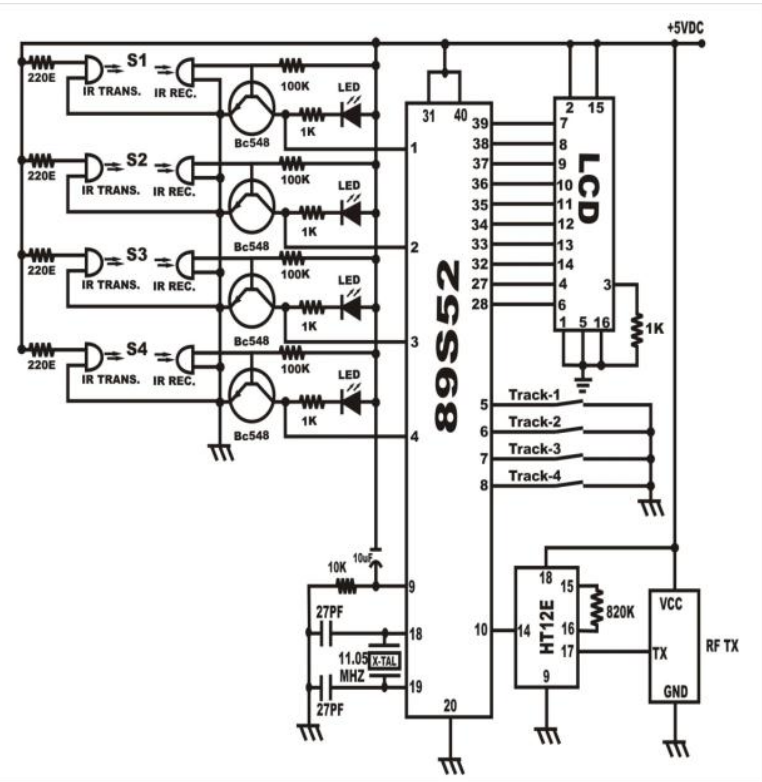

Fig. 7. Circuit Diagram

The proposed concept already started to design and we are excepting the output. In real time, it is not possible because we have to get the permission from the government side, so in demo model we are designing this concept. If we are getting the output as per our demo model then we planned to approach the government to implement this concept in real time systems. Above mentioned figures are the block diagram and circuit diagram of our demo model.

\section{Results And Discussions}

Our proposed design is facing a new challenge to further improve the reliability of rail testing techniques, while seeking for new and emerging technologies in automation engines that aid the detection of rail defects. Automation Engine (AE) will be done using RF Transmitter and Receiver, CAN Controller, GSM, GPS, LCD or Alarm. This proposal gives better accuracy, very fastest operation in Real-time where the human life is very important. The main result of our design is to detect the crack using advanced technologies so as to avoid human error. The sample output of crack detection in our demo model is given below.

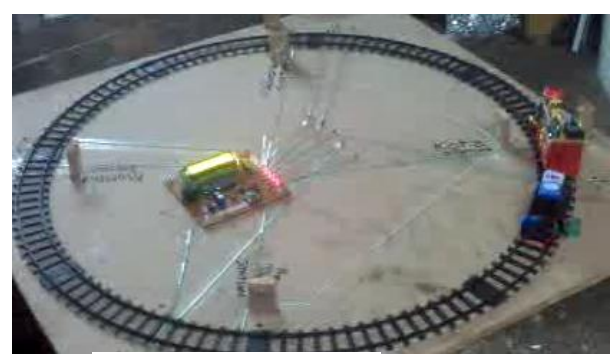

Fig. 8. Demo Output

\section{Conclusion}

From the monitoring data the following conclusions are drawn:

- In the railway track, cracks are occurring in two ways by natural and artificial

- The natural means (like high expansion due to heat, water floods)

- The artificial cracks are occurs due to antisocial elements (like terrorists, naxalites).

From our analysis these two cracks are occurring periodically, but apart from this cracks some other cracks also occurs due to soil condition, water leakage problem. As per our proposed model using this automated engine design in real time means we can able to easily avoid the accidents occur by Track side faults. 


\section{References}

[1] Avinash Vanimireddy, D.Aruna Kumari, Automatic Broken Track Detection Using LED-LDR Assembly, International Journal of Engineering Trends and Technology (IJETT) - Vol. 4, Issue 7- July 2013

[2] M. Kalaimathi, P. Ilakya \& E. Sathiavathy, Innovative Railway Track Surveying with Sensors and Controlled by Wireless Communication, ISSN (Print) : 2278-8948, Vol. 2, Issue 3, 2013

[3] S. Ramesh, S. Gobinathan, Railway Faults Tolerance Techniques using Wireless Sensor Networks, International Journal of electronics \& communication technology - Vol. 3, Issue 1, Jan.- March 2012

[4] Shaik Sulthan Basha, K Balakrishna, Robust railway crack detection theme (RRCDS) using LED-LDR assembly, International Journal of Reviews on Recent Electronics and Computer Science, August 2013/Vol. 1/Issue 4/425-429, ISSN 2321-5461

[5] Ramesh Shunmugam, Ramesh.K, Modeling and Detecting Damage in Rails \& Avoidance of Collision in the Tracks, International Journal of Scientific \& Engineering Research, Vol. 2, Issue 2, February-2011, ISSN : 2229-5518

[6] Stuart B Palmer, Steve Dixon, Rachel S Edwards and Xiaoming Jian, Transverse and longitudinal crack detection in the head of rail tracks using Rayleigh wave-like wideband guided ultrasonic waves

[7] Ch. Muneendra Rao, B. R. Bala Jaswanth, Crack Sensing Scheme in Rail Tracking System, International Journal of Engineering Research and Applications, ISSN : 2248-9622, Vol. 4, Issue 1( Version 2), January 2014

[8] http://www.atmel.in/Images/doc1919.pdf

[9] http://en.wikipedia.org/wiki/Global Positioning_System

[10] http://en.wikipedia.org/wiki/Liquid-crystal_display

[11] http://www.tc.gc.ca/media/documents/railsafety/technologies.pdf 